\title{
Neural Substrates of Contextual Interference during Motor Learning Support a Model of Active Preparation
}

\author{
Emily S. Cross, Paul J. Schmitt, and Scott T. Grafton
}

\begin{abstract}
When individuals acquire new skills, initial performance is typically better and tasks are judged to be easier when the tasks are segregated and practiced by block, compared to when different tasks are randomly intermixed in practice. However, subsequent skill retention is better for a randomly practiced group, an effect known as contextual interference (CI). The present study examined the neural substrates of CI using functional magnetic resonance imaging (fMRI). Individuals learned a set of three 4-element sequences with the left hand according to a block or random practice schedule. Behavioral retest for skill retention confirmed the presence of a typical CI effect with the random group outperforming the block group. Using a go/no-go fMRI paradigm, sequence preparation
\end{abstract}

\section{INTRODUCTION}

Contextual interference (CI) has been extensively examined in a variety of motor learning paradigms since this phenomenon was first proposed in 1972 (Battig, 1972) and experimentally demonstrated in 1979 (Shea \& Morgan, 1979). The CI effect is defined as the case when learning a set of task components with high levels of interference between the individual components leads to improved task retention and transfer, usually in the setting of poor performance during training (Battig, 1972). The effect is most commonly established by assigning participants to practice the set of task components in either a block or random schedule. Individuals who have been assigned to a random practice schedule in CI experiments commonly report that learning the components of a new skill with random practice (high $\mathrm{CI}$ ) is frustrating because performance improvements come so slowly over the course of practice. However, on retention tests, individuals from this group typically outperform fellow participants who were trained according to a block practice schedule (Figure 1). This effect has since been demonstrated in learning many physical skills including how to cartwheel (Smith, Gregory, \& Davies, 2003), swing a golf club (Brady, 1997; Goodwin \& Meeuwsen, 1996), tie knots (Ollis, Button, \& Fairweather,

Dartmouth College during the premovement study period was separated from movement execution. Imaging data for the two groups were compared for the first $1 / 3$ and final $1 / 3$ of training trials. Toward the end of training, behavioral performance between the two groups was similar, although the random group would later display a performance advantage on retention testing. During study time, the random group showed greater activity in sensorimotor and premotor regions compared to the block group. These areas are associated with motor preparation, sequencing, and response selection. This pattern of recruitment is consistent with the hypothesis that CI benefits in a sequencing task are due to improved capacity to actively prepare motor responses.
2005), execute snowboarding turns (Smith, 2002), and roll a kayak (Smith \& Davies, 1995).

Consequently, the CI effect has received considerable attention from motor learning researchers interested in explaining why CI occurs (Lee \& Simon, 2004; Magill \& Hall, 1990). It is interesting to note that the presence of $\mathrm{CI}$ is not unique to the motor learning domain. Studies reporting the presence of a $\mathrm{CI}$ effect when learning foreign language vocabulary (Schneider, Healy, Ericsson, \& Bourne, 1995) and when learning high-level cognitive tasks, such as how to troubleshoot a complex system (de Croock, van Merrienboer, \& Paas, 1998), demonstrate that $\mathrm{CI}$ is a more general phenomenon that applies to a variety of learning contexts. The paradox of increased interference, worse performance, and slower acquisition at initial training leading to enhanced performance after a delay has motivated researchers to evaluate participants' performance on individual task components in order to determine why the CI effect is manifest.

In one such study designed to characterize the component processes underlying the CI phenomenon, Immink and Wright (1998) conducted three experiments measuring performance on a simple keypress task when the amount of time allocated for studying the sequences was manipulated. In the first two experiments, Immink and Wright allowed participants to spend as much study time to each trial of the task as they wished. In the third experiment, all participants were allocated either 1 or $2 \mathrm{sec}$ 
Figure 1. Representation of the contextual interference (CI) effect. During block practice, intratask (within task) processing is more important than intertask (between task) processing for learning new tasks. For individuals learning a task according to a random practice schedule, theorists propose that both intratask and intertask processing are used. When individuals have experienced a high level of contextual interference (as in the random practice schedule), their performance is poor (compared to those who do not experience contextual inference) during initial task acquisition, but is better than those who did not experience contextual interference on later retention and transfer tasks.

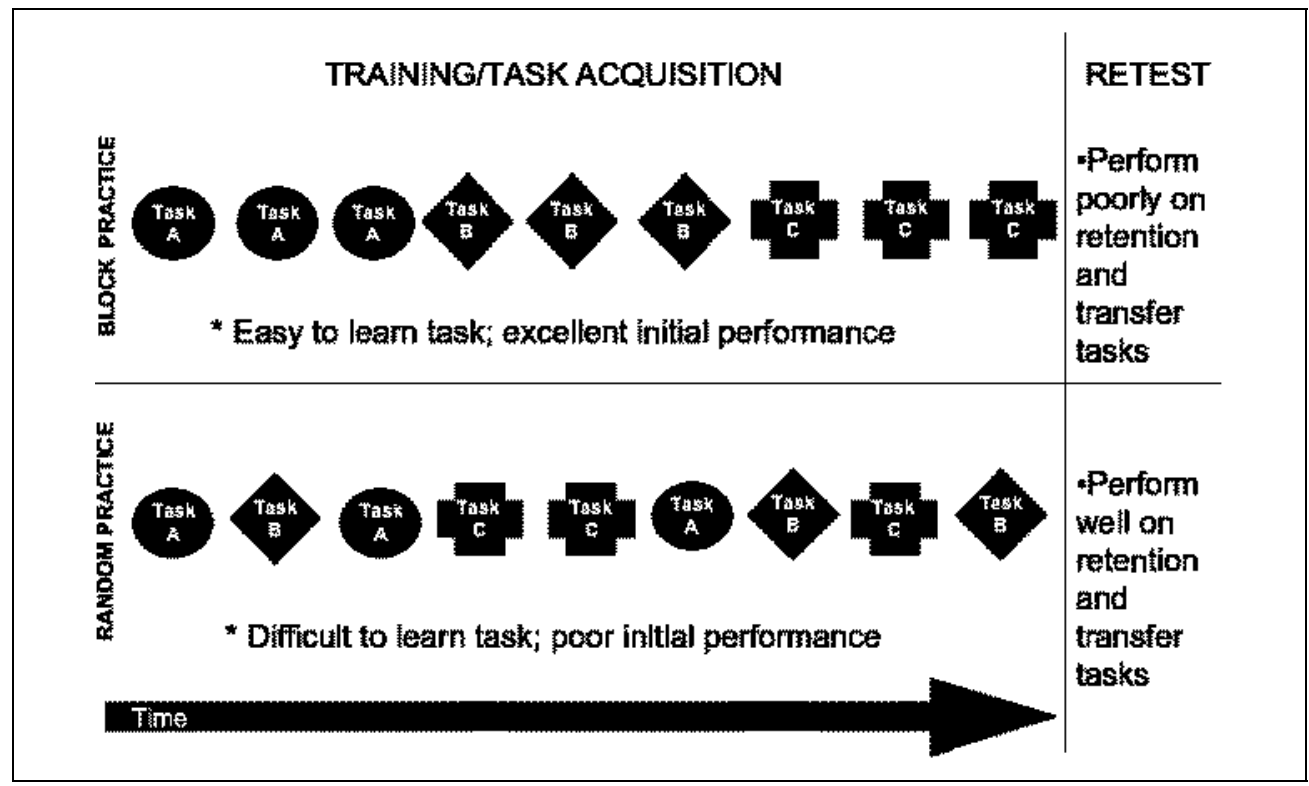

of study time, predetermined for each trial by the experimenter. One theory behind the CI effect posits that during random practice, task information must continually be reconstructed between each trial, and this process places high demand on attentional or preparatory processes immediately before task execution (Li \& Wright, 2000). If such a hypothesis was true, then participants in the random schedule should take more time to "reconstruct" what is required of them for each trial than participants in the block schedule, and it is this deeper level of reconstruction that leads to enhanced retention.

Immink and Wright reported that random-schedule participants in the first two experiments did indeed require greater study time, and in turn, demonstrated enhanced performance on the retention task one day later. A curious finding reported by these authors was a lack of an initial acquisition and performance benefit by the individuals assigned to the block practice schedule when their random-practice counterparts were given sufficient time to study and plan the movements to be executed. The third experiment demonstrated that an imposed limit on study time forced participants in the random practice condition to use movement time to continue to plan their responses; a direct linear relationship was present, indicating that in the random condition, participants' movement times increased as study times decreased. This pattern was not found for participants in the block practice condition; their study time spill-over into movement time only lasted for the first few trials, and then they were consistently faster than the random practice participants to execute the sequences.

Taken together, the studies by Immink and Wright (1998) and others (Li \& Wright, 2000; Lee \& Magill, 1983; Jacoby, 1978) suggest that the performance benefits at retention testing for participants who train under a random schedule are due to more time spent reconstructing the component movements during the training period. Two critical questions are what, precisely, is occurring during the reconstruction process and what neural substrates support this process. A plausible hypothesis is that individuals in the random group engage in more or deeper retrieval, planning, and preparation while reconstructing the cued movements. The present study aims to address this by measuring neural activity associated with both movement preparation and execution when learning a novel motor task according to either blocked or random conditions. To do this, the approach of Immink and Wright was used to develop a functional magnetic resonance imaging (fMRI) experiment. We used the version of their experiment where participants have unlimited study time to mentally rehearse and prepare any given movement sequence, which should ultimately lead to similar task performance between the two groups by the end of training but differential performance on retention testing. This is advantageous for the purposes of comparing the two groups because any difference in brain activity by the end of training should be related to the influence of the practice schedule on those neural systems that are modified by practice structure for the sequencing task and not on differences in performance confounds such as time spent preparing or executing a movement, or responding to an imperative cue to perform a movement.

If $\mathrm{CI}$ benefits in the random group are due in large part to more or deeper retrieval, planning, or preparation of the upcoming sequence, then differences between the two groups should be more pronounced during motor preparation rather than execution. Thus, 
it becomes particularly important to dissociate brain activity during planning and execution periods. This is difficult to do for single trials because of the temporal correlation from the hemodynamic response in blood oxygen-level-dependent (BOLD) MRI imaging. However, by using a go/no-go task, sequence preparation and sequence execution components could be decoupled (e.g., Johnson-Frey, Newman-Norlund, \& Grafton, 2005). We hypothesized that CI benefits for our particular skill would emerge in brain areas associated with sequence planning and movement preparation. An extensive behavioral and neuroimaging literature has characterized the features of learning a new sequential motor task, and at its core, motor learning entails the organization and execution of a selected action or series of actions (Rosenbaum, 1991; Keele, 1986). Of the abundant literature on the behavioral manifestations and neural substrates of sequence learning, most studies use serial reaction time (SRT) tasks to study elements of implicit and explicit sequence learning (for reviews, see Ashe, Lungu, Basford, \& Lu, 2006; Forkstam \& Petersson, 2005). In the SRT task, each element of a sequence is cued individually, with the cue also serving as an imperative to respond. Thus, there is no separation of preparation and execution. Although the present study did not use an SRT task, findings from the well-established literature on reconstructing motor sequences in terms of preparation and execution in response to symbolic cues can, nonetheless, help to generate plausible predictions for the current experiment. Converging evidence implicates the involvement of the dorsal premotor (PMd) cortex in response preparation in general as well as for sequence learning (Diedrichsen, Grafton, Albert, Hazeltine, \& Ivry, 2006; Kansaku et al., 2005; Bischoff-Grethe, Goedert, Willingham, \& Grafton, 2004; Diedrichsen, Werner, Schmidt, \& Trommershauser, 2004; Grafton, Hazeltine, \& Ivry, 1998, 2002; Toni, Ramnani, Josephs, Ashburner, \& Passingham, 2001; Toni, Rushworth, \& Passingham, 2001; Passingham, Toni, \& Rushworth, 2000; Jenkins, Brooks, Nixon, Frackowiak, \& Passingham, 1994). Other areas that facilitate sequence learning may be found on the superior and medial frontal gyri (SFG and MFG), which also help coordinate action selection when participants must change response sets (Rushworth, Hadland, Paus, \& Sipila, 2002), and also contribute to the coordination of smooth movement sequencing (Kennerley, Sakai, \& Rushworth, 2004; Rushworth, Walton, Kennerley, \& Bannerman, 2004). The involvement of the motor cortex (M1) has also been implicated in the storage of motor sequence information, particularly when the learning is more motoric than perceptual in nature. This has been demonstrated by extracellular recordings of single neurons in primary motor cortical areas of monkeys (Matsuzaka, Picard, \& Strick, 2007; Lu \& Ashe, 2005; Ben-Shaul et al., 2004) and in sequence consolidation in humans, shown with repetitive transcranial magnetic stimulation (rTMS) over M1 in humans
(Robertson, Press, \& Pascual-Leone, 2005; Muellbacher et al., 2002).

Heretofore, the CI effect has never been examined with functional neuroimaging, so specific predictions of CI-induced neural effects are difficult to make. However, based on the motor response preparation and sequence learning literature outlined above, we expect to see differences of neural activity at the end of training and as a function of practice schedule in the major brain areas commonly implicated in sequence learning, such as M1, SFG, and premotor cortical areas. If subjects are reconstructing the desired sequences during the study period because of the random training schedule, then we would predict greater activity in motor preparatory areas to manifest during the study period for this group.

\section{METHODS}

\section{Participants}

Of the 30 adult individuals from the local community who participated in this study, 15 were randomly assigned to the block practice condition and the other 15 received random practice. All participants had normal vision and no history of neurological or psychiatric disorders and provided written informed consent to participate in this study in a manner approved by the Committee for the Protection of Human Subjects at Dartmouth College. Two subjects from the random group were excluded because their behavioral response times were greater than 2 standard deviations above the random group mean for study time and movement time, and one subject from the block group was excluded because his mean neuroimaging findings were greater than 2 standard deviations below the mean activation pattern. Of the remaining 27 subjects ( 11 women, mean age $=21.25 \pm 3.1$ years, 13 in the random group and 14 in the block group), all were strongly right-hand dominant according to the Edinburgh Handedness Inventory (Oldfield, 1971). A subset of 15 participants (6 women; 7 from the random group and 8 from the block group) returned for a behavioral retest task several months (mean time since training $=3.69 \pm 2.9$ months) after initial scanning.

\section{Stimuli}

Stimuli for each participant were 3 of 22 possible fournumber sequences comprising the numbers 1 through 4 in all possible combinations, except where the numbers follow sequentially from each other (e.g., 1234 and 4321). In the behavioral retest, there was also a novel retest condition wherein participants performed three new sequences they did not train on during the initial training period in the scanner. 


\section{Behavioral Procedure}

Visual stimuli were presented and behavioral data were recorded on a Dell Optiplex GX1 desktop personal computer running Presentation software (Neurobehavioral Systems; www.neurobs.com). All stimuli were back projected from an Epson LCD projector (Model ELP-7000) onto an adjustable angled mirror mounted at the top of the head coil. Each subject positioned the four fingers of his or her left hand on the four light-sensitive response keys of a fiber-optic keypad. This apparatus was used to collect responses, which were recorded through a house-built data collection box. The left hand was used to increase the overall motor difficulty of the task, analogous to the approach of Immink and Wright (1998).

This study employed an event-related design to measure between-group brain activation differences when participants studied and when they performed movement sequences. Participants were instructed to perform each trial as quickly and as accurately as possible, and received feedback after each trial on the speed and accuracy of their performance (see Figure 2 for a representation of one trial sequence). Both correct and incorrect trials were included in the fMRI analysis, and no reliable differences were seen between the block and random practice groups in terms of task accuracy $(t=1.31, p=.20)$. Participants performed a total of 54 go trials and 54 no-go trials (18 for each of 3 number sequences) in either block or random order. Study time and movement time were subjectpaced, and participants spent between 21 and $28 \mathrm{~min}$ on the behavioral task while being scanned.

The behavioral retest was performed with the same equipment and stimuli as that used while training in the scanner. The only difference was that participants now

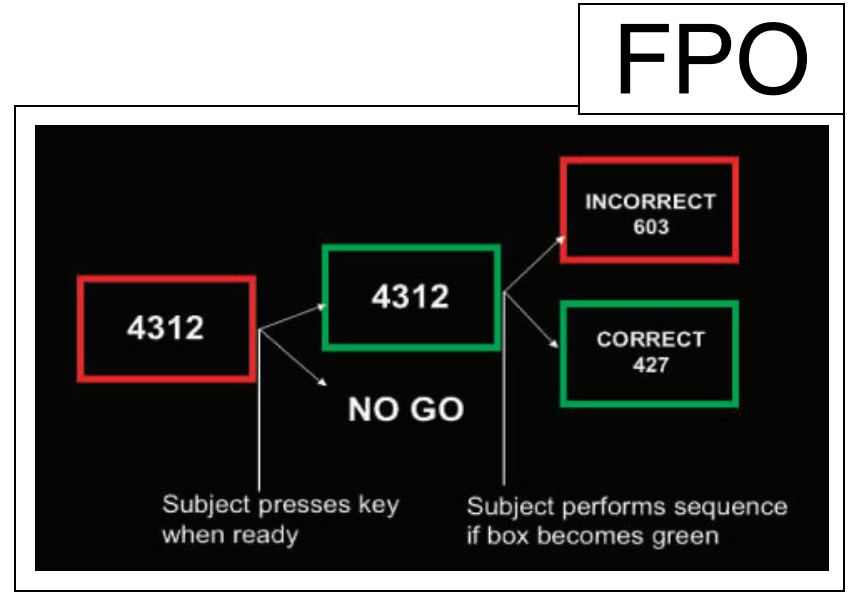

Figure 2. Representation of one trial sequence. The sequence to be performed is presented with a red box around it. Participants study the sequence for as long as they need to prepare the movement sequence, then press any key to signal their readiness. After a short delay, either the red box around the sequence will turn green, denoting that the subject should perform the sequence as quickly and accurately as possible, or the words "no go" will appear, and the subject rests until the next sequence appears. After completion of each go trial, participants receive feedback about their performance in terms of accuracy and the time it took to complete the sequence. performed the sequences on the $\mathrm{A}, \mathrm{S}, \mathrm{D}$, and $\mathrm{F}$ keys of standard PC keyboard with their left hand, instead of on a fiber-optic keypad. The letters on the keyboard were covered to eliminate any potential conflict between the letters and their assigned numbers for this particular task. In addition to performing the same three sequences they trained on in the scanner, participants performed three novel sequences in blocked order to test the transfer of procedural knowledge. This novel condition was included to test the robustness of the CI effect of our manipulation, as is routinely done in CI studies (Magill \& Hall, 1990; Lee, Magill, \& Weeks, 1985; Shea \& Morgan, 1979). All participants performed the behavioral retest trials in the same grouping; first nine trials presented in blocks of 3 , then nine trials presented randomly, and finally, nine trials of three novel sequences presented in blocks of 3 .

\section{Imaging Procedure}

Images were acquired with a 1.5-T General Electric Signa scanner using a standard birdcage head coil. Head movements were minimized with the use of a foam pillow and padding. Images were acquired continuously during functional scanning using a gradient-echo, echo-planar pulse sequence $(\mathrm{TR}=2.5 \mathrm{sec}$; $\mathrm{TE}=35 \mathrm{msec}$; flip angle $=90^{\circ}$; field of view $=24 \mathrm{~cm} ; 3.75 \times 3.75 \mathrm{~mm}$ in-plane resolution). The first four volumes of each functional run were discarded to allow for longitudinal magnetization to approach equilibrium, and then an additional 504-642 volumes of axial images were collected (depending on how long it took for each participant to complete all trials of the task), with 25 slices per TR (4.5 $\mathrm{mm}$ thickness, $1 \mathrm{~mm}$ gap) allowing whole brain coverage.

\section{Imaging Analyses}

Functional data were analyzed with Statistical Parametric Mapping software (SPM2, Wellcome Department of Cognitive Neurology, London, UK; Friston et al., 1998). For each functional run, data were realigned, unwarped, and normalized to the MNI template with a $2 \times 2 \times 2 \mathrm{~mm}$ resolution, which approximates Talairach \& Tournoux (1988) atlas space. A 6-mm smoothing kernel was applied to the normalized images. An individualized design matrix was generated and fitted for each subject incorporating task effects (modeled with a canonical hemodynamic response function; Friston et al., 1998) to compute parameter estimates (beta) and $t$-contrast images (containing weighted parameter estimates) for each comparison at each voxel. Study times for the 54 no-go trials and movement times for the 54 go trials were separately modeled. Subjects' individual study times for each no-go trial were explicitly modeled as events with subject-specified durations, and movement times were modeled as events. Both of these parameters 
were split into three sets, comprising the first 18 trials, the second 18 trials, and the last 18 trials. This was done in order to examine differences in early versus late imaging data. This also enabled us to examine independently brain activity during movement preparation at the end of training (the last 18 trials), when behavioral performance of the two groups was statistically indistinguishable. The general patterns of neural activation associated with movement and study time were also evaluated with both groups collapsed together across all 54 trials. The main effects of movement and study time were used to create two masks with which to interrogate brain regions for between-group differences during the early versus late between-group interactions. This was done to reduce the search volume and decrease the rate of type II error. A representative firstlevel design matrix is illustrated in Figure 3. Results from the contrasts for individual subjects were submitted to a random effects group-level analyses, wherein differences between the two experimental groups were masked by the main effects of study time or movement time, and were evaluated at the $p<.005$ level, uncorrected, with a minimum cluster size of $64^{3} \mathrm{~mm}$. Additionally, regionof-interest (ROI) analyses were performed on selected brain regions revealed by the repeated measures interactions between group (block vs. random) and training time (early vs. late).

\section{RESULTS}

\section{Behavioral Results: Training}

Participants in the block group studied less than participants in the random group (Figure 4). A 2 (group: block

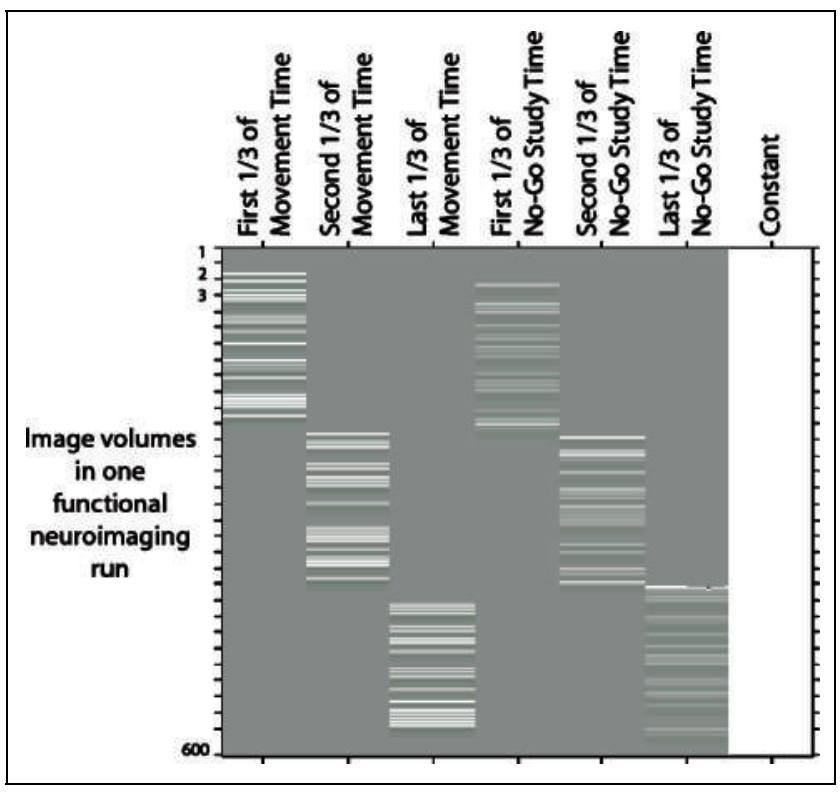

Figure 3. Representative design matrix for first-level contrasts. During each imaging run, both movement execution and movement preparation trials were grouped into thirds.

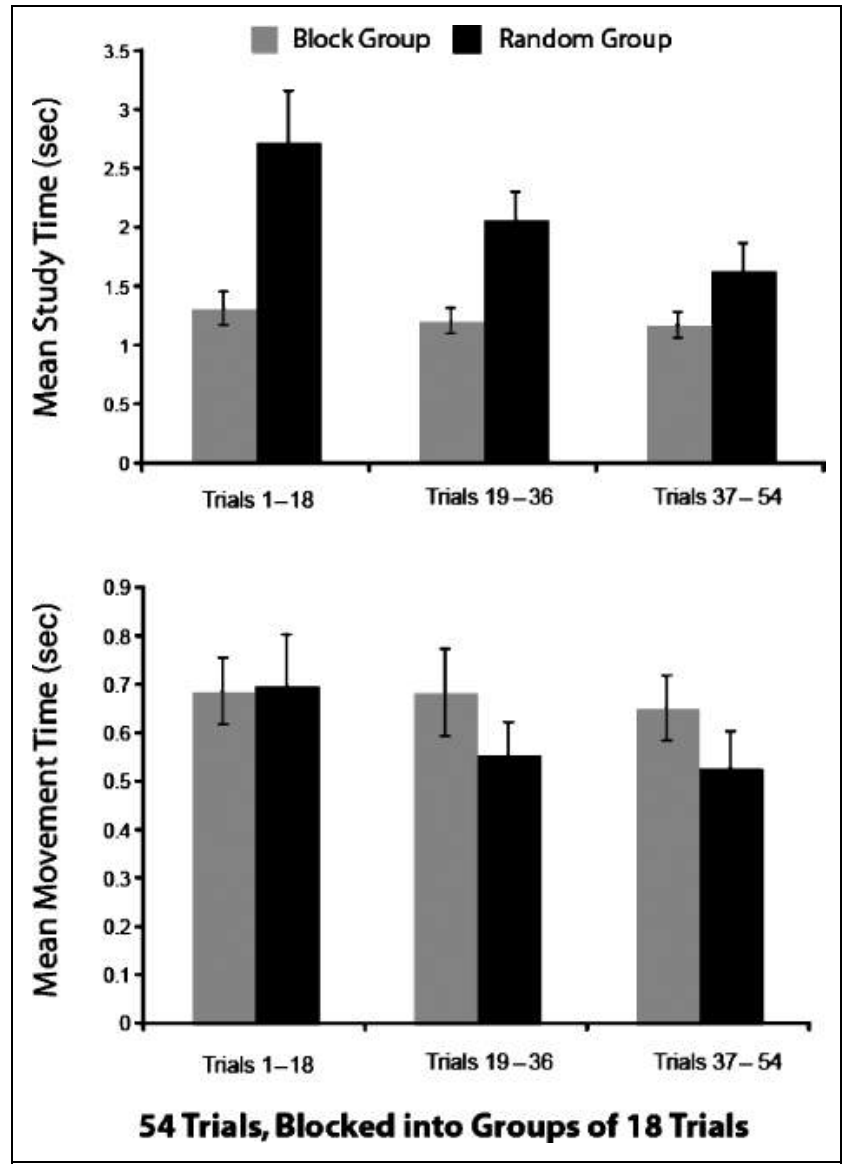

Figure 4. Mean movement and study times (in seconds) for participants in the block and random groups at time of training. Error bars represent standard error of the mean. For viewing purposes, the 54 trials in the task are binned into groups of 18 trials. Imaging analyses focus on changes in brain activation between early practice (trials 1-18) and late practice (trials 37-54).

or random) $\times 3$ (session blocked into thirds: early trials (1st third), middle trials (2nd third), and late trials (final third)) repeated measures analysis of variance (ANOVA) revealed two significant main effects and an interaction. Participants studied less as they advanced from Session 1 through Session 3, $F(1.21,30.2)=13.78, p<.0001$. There was also a significant main effect of group, with the block group studying less than the random group across all sessions, $F(1,25)=14.63, p=.001$. A Session $\times$ Group interaction was observed with this analysis as well, with study times converging as the sessions progressed, $F(1.21,30.2)=8.16, p=.005$. A separate paired $t$-test analysis of study times for go trials and no-go trials confirmed that no reliable differences existed between study times for go trials $(M=1.78 \mathrm{sec})$ and no-go trials $(M=1.78, t=-0.008, p=.99)$.

In terms of movement execution, a 2 (group) $\times 3$ (session thirds) repeated measures ANOVA run on these data revealed one main effect and an interaction. Regardless of training group, participants performed the sequences more quickly as time progressed, $F(2,50)=$ 
$6.91, p=.002$. Additionally, the Session $\times$ Group interaction indicated that participants in the random training group showed greater decreases in movement time across sessions than did the block group, $F(2,50)=$ 3.91, $p=.03$.

For imaging analyses, we were interested in assessing between-group differences in neural activity and how these differences might change between early and late practice during the experimental session. However, the between-group differences in the behavioral data that are characteristic of the $\mathrm{CI}$ effect present a challenge for fMRI data interpretation. Potential difficulties in imaging data interpretation can arise when behavioral performance is not equivalent. In order to determine the degree of inequality of the data of interest, we ran separate independent-samples $t$ tests on the study and movement behavioral data of the two groups for the early (first $1 / 3$; trials $1-18$ ) and late (last $1 / 3$; trials $37-$ 54) training trials. Unsurprisingly, an independent $t$ test for differences between groups for early study time revealed a significant effect, with the random group studying longer than the block group, $t(25)=-4.22, p<.001$. The same test evaluating differences between the two groups during the late trials revealed no significant differences between the block and random groups, $t(25)=$ $-1.89, p=.07$. For movement time data, no betweengroups differences were found for early, $t(25)=-0.102$, $p=.92$ or late movement times, $t(25)=1.26, p=.22$. The finding of unequal study time during early training needs to be kept in mind for the subsequent interpretation of the imaging data interactions that characterize differences between groups as a function of how much training they had experienced.

\section{Behavioral Results: Retest}

In line with the classic CI effect, participants in the random group performed generally better than participants in the block group (Figure 5). No between-group differences in accuracy were observed, with both groups performing all retest sequence types at approximately 90\% accuracy. Differences between the block and random groups in study and movement times for the keypress sequences were tested with 2 (block or random group) $\times 3$ (retest sequence type; block, random, or novel block) repeated measures ANOVAs. Analysis of study time data revealed one main effect and one interaction. A main effect of sequence type showed that participants studied the most for the block sequences (mean $=2.05 \mathrm{sec}$ ), an intermediate amount for random sequences $(M=1.74)$, and the least for the novel sequences $(M=1.55) ; F(2,26)=9.49, p=.001$. We interpret this to be simply an effect of time, as all participants performed the retest trials in the same sequence (first block, then random, then novel). An interaction between sequence type and group also emerged, with participants in the random learning group studying the

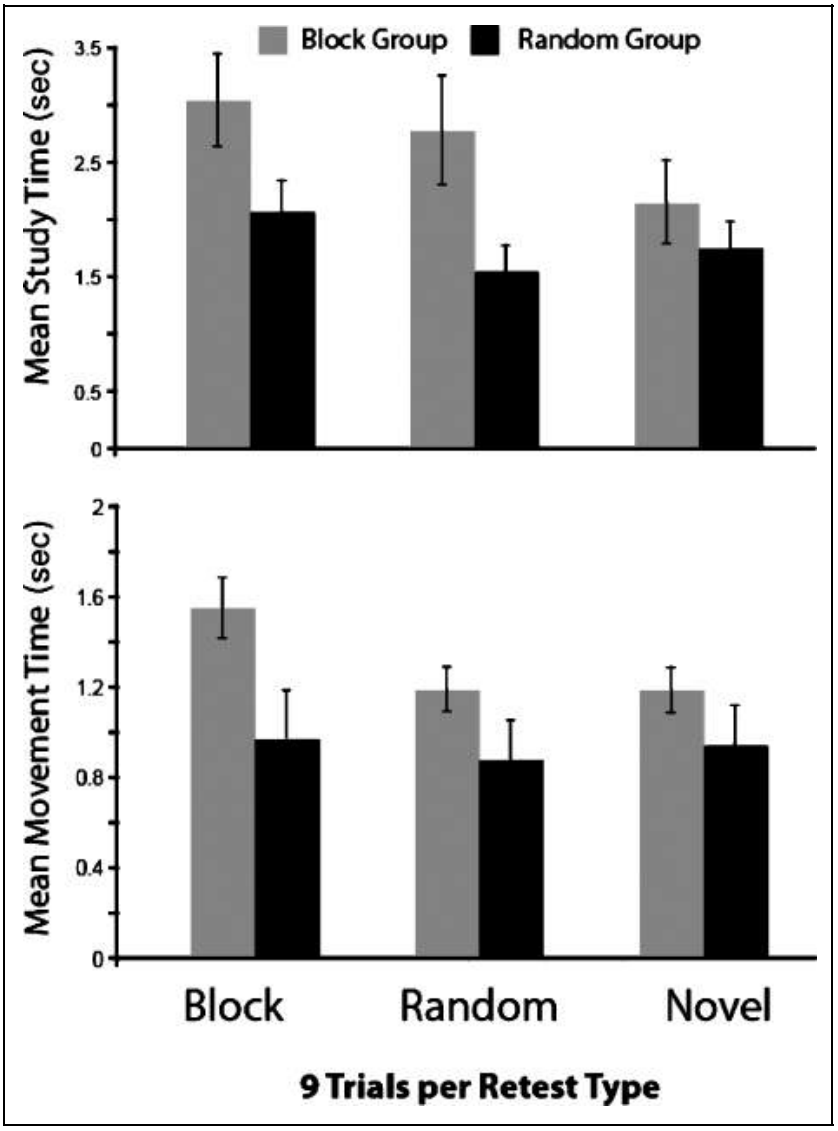

Figure 5. Mean movement and study times for the subgroup of participants who participated in the behavioral retest. Error bars represent standard error of the mean. For viewing purposes, the 27 trials in the entire retest task are binned into groups of 9 trials, based on the sequence type (block, random, or novel).

least amount for random sequences, thereby demonstrating that this group experienced the greatest performance improvements for sequences presented in same manner as they were learned, $F(2,26)=4.58, p=.02$. This finding is precisely in line with Shea \& Morgan's (1979) original report demonstrating the CI effect on later task performance. Although the main effect of group was not significant in this analysis, $F(1,13)=3.36$, $p=.09$, independent-samples $t$ tests revealed a difference between the block and random groups for study times for the blocked sequences that nearly reached statistical significance, $t(11.8)=2, p=.06$, and a significant difference in study times for the randomly presented sequences, $t(9.78)=2.35, p=.04$. Analysis of movement time data revealed a significant main effect of sequence type, with performance being slowest for the block movement sequences, again most likely due to an order effect, $F(1.25,16.27)=5.58, p=.025$. The test of between-subjects effects revealed a trend of a difference in movement times between the groups, with the block group moving more slowly than the random group, $F(1,13)=3.76, p=.07$. Independent-samples $t$ tests of movement time differences between the block and 
random groups for the three sequence types revealed a significant difference in movement times for the blocked sequences only, $t(13)=2.36, p=.03$.

\section{fMRI Results}

\section{Main Effects of Tasks}

The present study tested for differences in neural activity while preparing and executing simple keypress sequences between participants who learned the sequences according to a random practice schedule with those who learned according to a block practice schedule. Before interrogating the data for any between-group differences, we first identified common neural substrates among all participants that are involved in movement preparation and movement execution. We evaluated participants' study time and movement time data from the entire training session, collapsed across block and random practice groups. The findings from these analyses are displayed in Figure 6. Study time engaged areas that are classically associated with movement preparation, including the dorsal and ventral premotor cortex, the supplementary motor area, the inferior and superior parietal lobules, and the ventral prefrontal areas (see Table 1 for a complete listing of brain areas). Note that this contrast only calculates effects of study times from the no-go trials to avoid contamination by subsequent actual finger movements. Movement execution engaged brain regions associated with movement execution included the ipsilateral anterior cerebellum, the contralateral primary sensorimotor cortex, the basal ganglia, and the supplementary motor area (see Table 2 for a complete listing of brain areas). The imaging findings for movement execution also detected bilateral activity in the supplementary motor area, as well as the motor and premotor cortical areas. This pattern of bilateral activity during movement execution is supported by the literature on the lateralization of neural responses during sequential finger movements, which shows pronounced bilateral activation of these areas during left-handed keypresses (e.g., Kansaku et al., 2005).

\section{Group by Time Interactions}

This analysis compared areas of activation that differ between the block and random groups as a function of time when subjects prepared the to-be-performed sequences. All comparisons are restricted to the common network of brain regions identified involved in movement preparation (Figure 6A). The independentsamples $t$ test investigating the interaction between time (early vs. late) and group (random vs. block) for increases in random group and decreases in the block group revealed four cortical areas that demonstrated this pattern (Figure 7 and Table 3). Activity in the right lateral occipital cortex (in MNI coordinates: $x=46, y=$ $-84, z=-4)$, left primary motor cortex $(x=-22$, $y=-30, z=48)$, right precentral cortex $(x=52, y=$ $-12, z=58)$, and right paracentral lobule $(x=6$, $y=-44, z=64$ ) was greater during the final one third of trials at the end of training than during the first one third of training in the random group. Less activity was seen in these areas at the end of training than in the beginning of training in the block group. The inverse contrast, exploring areas demonstrating greater activity at the end of training for the block group and decreased activity at the end of training for the random group, revealed two brain regions (Figure 8 and Table 3 ). These regions were located in the right lateral cerebellum $(x=$ $38, y=-54, z=-44)$ and in the left parahippocampal gyrus $(x=-32, y=-42, z=-4)$.

One additional analysis was run with the study time data to address the potential confound of the random group spending a greater amount of time studying the keypress sequences (illustrated in Figure 4 and presented in the behavioral results above). This was done because the greater amount of time spent studying by the random group can potentially complicate the interpretation of imaging data, as some differences in neural
Figure 6. Activation patterns for preparation of keypress sequences (Study Time, A) and of movement execution (Movement Time, B), collapsed across all participants, for all of training, regardless of practice structure. Image left is the left side of the brain. Threshold is $p<.005$, uncorrected, with an extent threshold of 5 voxels. Statistical images are superimposed on a mean anatomic image of the study population.

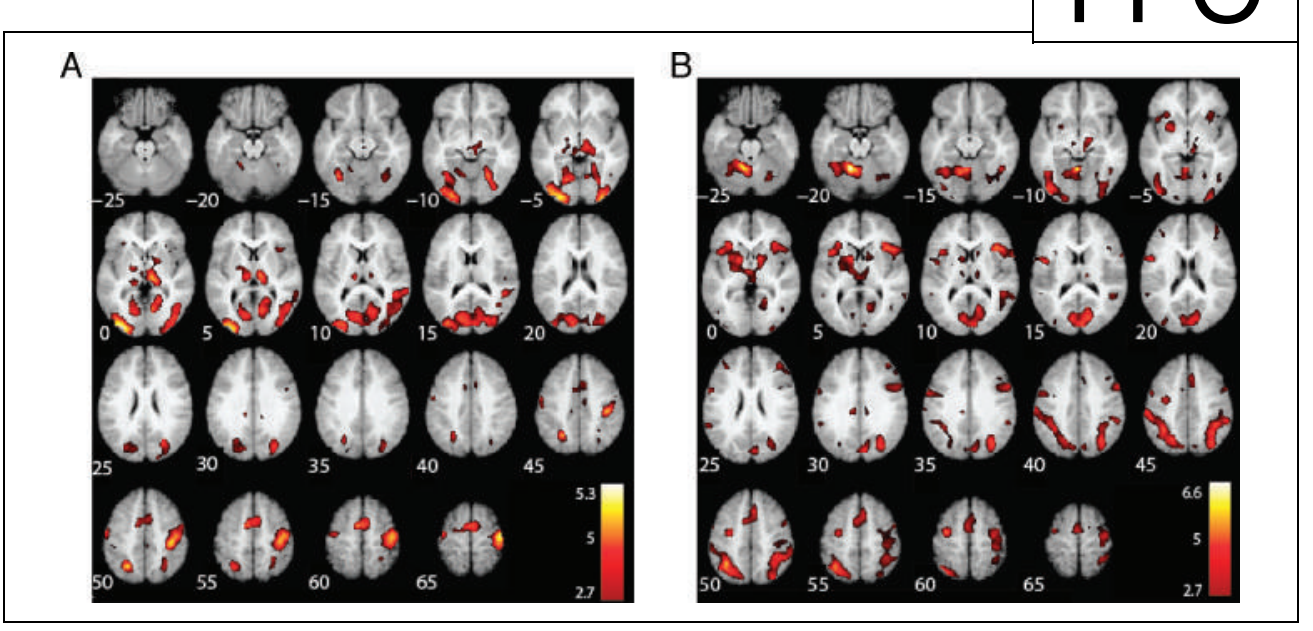


Table 1. Regions Showing Activity during Movement Preparation, Collapsed across Groups

\begin{tabular}{|c|c|c|c|c|c|c|c|}
\hline \multirow[b]{2}{*}{ Region } & \multirow[b]{2}{*}{$B A$} & \multicolumn{3}{|c|}{ MNI Coordinates } & \multirow[b]{2}{*}{ Functional Name } & \multirow[b]{2}{*}{$t$} & \multirow[b]{2}{*}{$p$} \\
\hline & & $x$ & $y$ & $z$ & & & \\
\hline L Inferior occipital gyrus & 18 & -34 & -94 & 0 & Visual association cortex & 5.28 & $<.0001$ \\
\hline R Postcentral gyrus & 6 & 42 & -18 & 68 & Dorsal premotor cortex (PMd) & 5.21 & $<.0001$ \\
\hline L Superior parietal lobule & 7 & -24 & -60 & 48 & SPL & 4.76 & $<.0001$ \\
\hline R Postcentral gyrus & 3 & 38 & -28 & 48 & & 4.55 & $<.0001$ \\
\hline R Occipital cortex & 18 & 32 & -96 & -6 & & 4.50 & $<.0001$ \\
\hline $\mathrm{R}$ Thalamus & & 10 & -22 & 0 & & 4.38 & $<.0001$ \\
\hline $\mathrm{R}$ Precentral gyrus & 4 & 44 & -16 & 52 & & 4.33 & $<.0001$ \\
\hline L Superior frontal gyrus & 6 & -10 & -2 & 56 & SMA & 4.16 & $<.0001$ \\
\hline R Superior frontal gyrus & 6 & 2 & -2 & 62 & SMA & 3.96 & $<.0001$ \\
\hline L Thalamus & & -10 & -20 & 8 & & 3.78 & $<.0001$ \\
\hline R Superior frontal gyrus & 6 & 6 & -6 & 68 & SMA & 3.76 & $<.0001$ \\
\hline L Precentral gyrus & 4 & -38 & -14 & 62 & Premotor cortex & 3.67 & .001 \\
\hline L Precentral gyrus & 4 & -56 & -10 & 50 & Premotor cortex & 3.62 & .001 \\
\hline L Globus pallidus & & -18 & -10 & -2 & & 3.51 & .001 \\
\hline R Putamen & & 18 & 2 & 0 & & 3.34 & .001 \\
\hline $\mathrm{R}$ Insula & 13 & 38 & 18 & 6 & & 3.16 & .002 \\
\hline R Superior frontal gyrus & 6 & 22 & -16 & 74 & & 3.11 & .002 \\
\hline L Inferior parietal lobule & 3 & -44 & -30 & 66 & IPL & 3.04 & .003 \\
\hline L Posterior cingulate cortex & 23 & -12 & -30 & 30 & & 3.03 & .003 \\
\hline L Posterior cingulate cortex & 31 & -12 & -26 & 46 & & 3.00 & .003 \\
\hline $\mathrm{R}$ Anterior cingulate cortex & 24 & 6 & -14 & 46 & & 2.96 & .003 \\
\hline L Ventrolateral prefrontal cortex & 45 & -30 & 26 & 0 & VLPFC & 2.96 & .003 \\
\hline $\mathrm{R}$ Inferior frontal gyrus & 45 & 46 & 22 & 2 & & 2.92 & .002 \\
\hline R Pons & & 2 & -28 & -48 & & 2.90 & .004 \\
\hline L Superior temporal gyrus & 22 & -54 & -44 & 8 & & 2.90 & .004 \\
\hline L Paracentral lobule & 7 & -4 & -32 & 50 & & 2.87 & .004 \\
\hline
\end{tabular}

Significance at all sites was tested by an independent-samples $t$ test on beta values averaged over each voxel in the cluster, uncorrected at $p<.005$, with a 5-voxel threshold.

activity between the groups might possibly be an artifact of significantly different amounts of time spent engaging in a particular process, not just different areas of activation related to training structure. The final betweengroups analysis was run on only the final one third of study time trials, when study times between groups were no longer statistically distinguishable $(p>.05)$. In this independent-samples $t$ test on imaging data, greater activity was observed in the left superior parietal cortex $(x=-34, y=-38, z=72 ; t=2.99, p=.001)$ and the left primary sensorimotor cortex $(x=-22, y=$ $-30, z=46 ; t=2.68, p=.004)$ in the random group compared to the block group. The complementary con- trast measuring activity that was greater in the block group than the random group for study time did not reveal any suprathreshold clusters (all $p$ values $>$.005).

We then tested for differences in neural activity over time between the two groups when executing the motor sequences, restricted to those regions identified to be a common network for movement execution across all participants (Figure 6B). This interaction identifies areas showing a differential response between early and late movement periods and between groups. Two regions showed decreased responses in the block group and increased responses in the random group across time (Figure 9 and Table 4). These regions were found 
Table 2. Regions Showing Activity during Movement Execution, Collapsed across Groups

\begin{tabular}{|c|c|c|c|c|c|c|c|}
\hline \multirow[b]{2}{*}{ Region } & \multirow[b]{2}{*}{$B A$} & \multicolumn{3}{|c|}{ MNI Coordinates } & \multirow[b]{2}{*}{ Functional Name } & \multirow[b]{2}{*}{$t$} & \multirow[b]{2}{*}{$p$} \\
\hline & & $x$ & $y$ & $z$ & & & \\
\hline L Cerebellum & & -12 & -52 & -22 & & 6.62 & $<.0001$ \\
\hline L Cerebellum & & -2 & -56 & -10 & & 5.92 & $<.0001$ \\
\hline L Intraparietal sulcus & 7 & -34 & -56 & 52 & IPS & 5.29 & $<.0001$ \\
\hline R Inferior frontal gyrus & 46 & 40 & 20 & 8 & DLPFC & 4.94 & $<.0001$ \\
\hline R Inferior parietal lobule & 40 & 42 & -44 & 46 & IPL & 4.67 & $<.0001$ \\
\hline R Primary visual cortex & 17 & 14 & -80 & 16 & V3 & 4.66 & $<.0001$ \\
\hline L Globus pallidus & & -14 & 4 & 2 & & 4.64 & $<.0001$ \\
\hline L Middle frontal gyrus & 6 & -32 & -10 & 60 & MFG & 4.62 & $<.0001$ \\
\hline R Inferior temporal gyrus & 20 & 38 & -48 & -36 & & 4.55 & $<.0001$ \\
\hline R Inferior parietal lobule & 40 & 34 & -72 & 48 & IPL & 4.55 & $<.0001$ \\
\hline L Inferior occipital gyrus & 18 & -42 & -86 & -8 & & 4.45 & $<.0001$ \\
\hline R Angular gyrus & 39 & 32 & -72 & 34 & & 4.44 & $<.0001$ \\
\hline L Anterior insula & 13 & -30 & 24 & 2 & & 4.33 & $<.0001$ \\
\hline L Inferior frontal gyrus & 44 & -48 & 2 & 16 & & 4.26 & $<.0001$ \\
\hline L Insula & 13 & -40 & 14 & 4 & & 4.21 & $<.0001$ \\
\hline R Middle frontal gyrus & 9 & 58 & 10 & 40 & MFG & 4.08 & $<.0001$ \\
\hline L Precentral gyrus & 4 & -30 & -12 & 50 & M1 & 4.01 & $<.0001$ \\
\hline R Inferior frontal gyrus & 44 & 64 & 10 & 12 & & 3.92 & $<.0001$ \\
\hline L Posterior cingulate cortex & 23 & -10 & -24 & 32 & & 3.79 & $<.0001$ \\
\hline R Superior frontal gyrus & 6 & 6 & 20 & 50 & Pre-SMA & 3.77 & $<.0001$ \\
\hline L Superior frontal gyrus & 6 & -2 & -6 & 64 & Caudal SMA (SMAc) & 3.69 & .001 \\
\hline L Superior frontal gyrus & 6 & -6 & 6 & 54 & Pre-SMA & 3.66 & .001 \\
\hline R Middle frontal gyrus & 46 & 42 & 42 & 24 & & 3.59 & .001 \\
\hline R Middle frontal gyrus & 6 & 50 & 0 & 54 & & 3.35 & .001 \\
\hline L Middle frontal gyrus & 46 & -34 & 38 & 24 & & 3.29 & .001 \\
\hline L Superior frontal gyrus & 6 & 18 & -4 & 68 & Dorsal premotor cortex (PMd) & 3.28 & .001 \\
\hline R Middle frontal gyrus & 9 & 42 & 22 & 38 & & 3.18 & .002 \\
\hline L Superior occipital gyrus & 19 & -32 & -84 & 20 & & 3.15 & .002 \\
\hline R Cingulate gyrus & 31 & 12 & -26 & 30 & Cingulate motor area (CMA) & 3.12 & .002 \\
\hline R Middle frontal gyrus & 46 & 52 & 32 & 26 & & 3.11 & .002 \\
\hline L Middle frontal gyrus & 10 & -40 & 46 & 22 & & 3.00 & .003 \\
\hline R Frontal operculum & 44 & 48 & 4 & 4 & & 2.94 & .003 \\
\hline L Thalamus & & -8 & -20 & -10 & & 2.94 & .003 \\
\hline $\mathrm{R}$ Precentral gyrus & 4 & 24 & -16 & 72 & M1 & 2.88 & .004 \\
\hline
\end{tabular}

Significance at all sites was tested by an independent-samples $t$ test on beta values averaged over each voxel in the cluster, uncorrected at $p<.005$, with a 5-voxel threshold. 


\section{FPO}

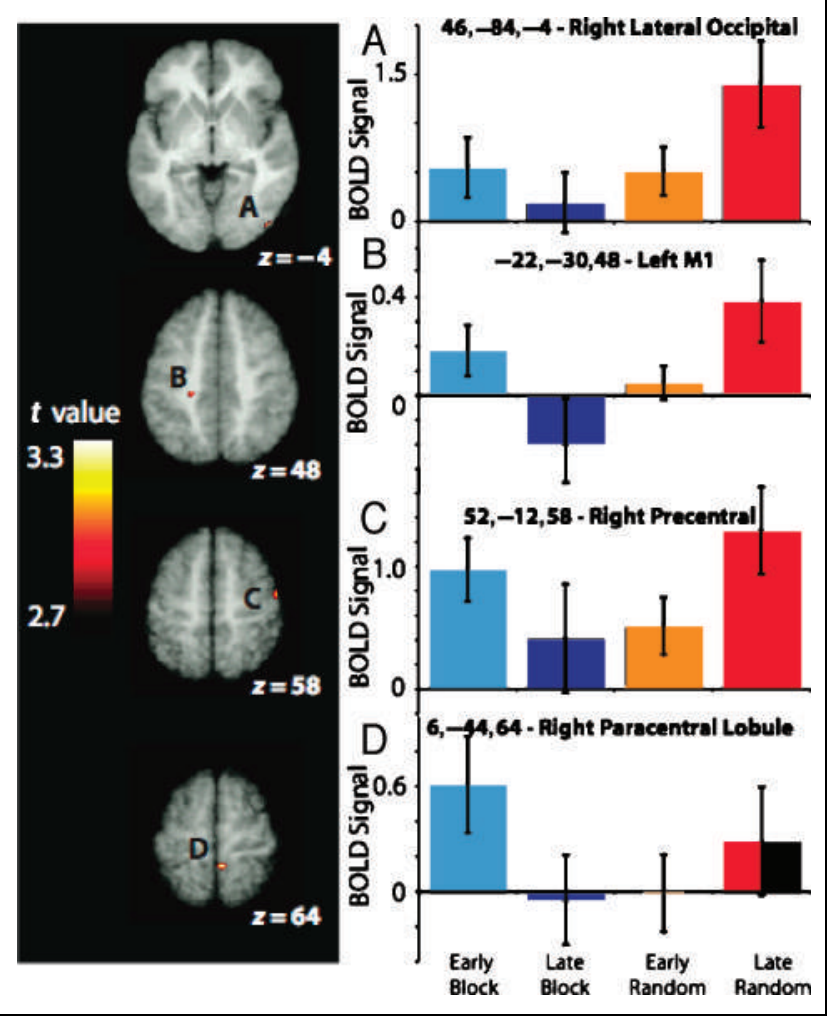

Figure 7. Group differences of motor preparation-interaction between increasing signal within the random group and decreasing signal within the block group. Differences were calculated from the first and the final $1 / 3$ of training trials, and are masked by regions that are active in all participants during study time (Figure 5A). The left image is the left side of the brain. Threshold is $p<.005$, uncorrected, threshold $>3$ voxels. Statistical images are superimposed on a mean anatomic image of the study population.

in the right SFG ( $x=26, y=60, z=24)$ and the left MFG $(x=-42, y=18, z=50)$. The inverse contrast revealed a large network of areas whose activity increased across time in the block group and decreased across time in the random group (Figure 10 and Table 4). For purposes of discussion, we have chosen to focus on a subset of four of the most significantly active regions, which include the right midline cerebellum $(x=$ $12, y=-44, z=-36)$, left insula $(x=-40, y=-18$, $z=12)$, left SFG $(x=-26, y=-4, z=56)$, and a region within the left superior parietal lobe $(x=-16, y=-15$, $z=64)$.

\section{DISCUSSION}

To our knowledge, this is the first functional imaging study to investigate the neural underpinnings of the CI effect. The present results extend past work on CI by characterizing potential neural substrates that might contribute to improved behavioral performance on retention testing after seemingly worse initial performance. The task structure was similar to a previous experiment by Immink and Wright (1998) and the current behavioral data echo their findings and demonstrate that the CI can be identified when subjects are trained and tested in the MRI scanner. Even in this complex training environment, the random group performed worse than the block group during training, and then outperformed the block group on the retention task. The data presented here also extend the idea proposed by Immink and Wright that individuals engaged in random practice simply need more time to plan their movements. The underlying assumption made by these authors is that random and blocked practice groups engage in the same planning and execution processes, but differ in the implementation of these processes across time. Our data show that not only do individuals in the random practice condition take more time to prepare upcoming movements, but they also recruit different brain regions than the block group during movement planning and execution.

Table 3. Regions Showing Greater Activity in the Between-Group Comparisons for Study Time

\begin{tabular}{|c|c|c|c|c|c|c|c|}
\hline \multirow[b]{2}{*}{ Region } & \multirow[b]{2}{*}{$B A$} & \multicolumn{3}{|c|}{ MNI Coordinates } & \multirow[b]{2}{*}{ Functional Name } & \multirow[b]{2}{*}{$t$} & \multirow[b]{2}{*}{$p$} \\
\hline & & $x$ & $y$ & $z$ & & & \\
\hline \multicolumn{8}{|c|}{ Study Time: Random Increasing (Early $>$ Late) $>$ Block Increasing (Early $>$ Late) } \\
\hline R Paracentral lobule & 5 & 6 & -44 & 64 & & 3.21 & .002 \\
\hline R Lateral occipital & $18 / 19$ & 46 & -84 & -4 & & 3.03 & .003 \\
\hline $\mathrm{R}$ Precentral gyrus & 6 & 52 & -12 & 58 & & 3.02 & .003 \\
\hline L Precentral sulcus & 4 & -22 & -30 & 48 & M1 & 3.00 & .003 \\
\hline \multicolumn{8}{|c|}{ Study Time: Block Increasing (Early $>$ Late) $>$ Random Increasing (Early $>$ Late) } \\
\hline R Cerebellum & & 38 & -54 & -44 & & 3.98 & $<.001$ \\
\hline L Parahippocampal gyrus & $35 / 36$ & -32 & -42 & -4 & & 2.78 & .003 \\
\hline
\end{tabular}

Significance at all sites was tested by an independent-samples $t$ test on beta values averaged over each voxel in the cluster, uncorrected at $p<.005$. 
Figure 8. Group differences of motor preparationinteraction between increasing signal within the block group and decreasing signal within the random group. Differences were calculated from the first and the final $1 / 3$ of training trials, and are masked by regions that are active in all participants during study time (Figure 5A). The left image is the left side of the brain. Threshold is $p<.005$, uncorrected, threshold $>$ 3 voxels. Statistical images are superimposed on a mean anatomic image of the study population.

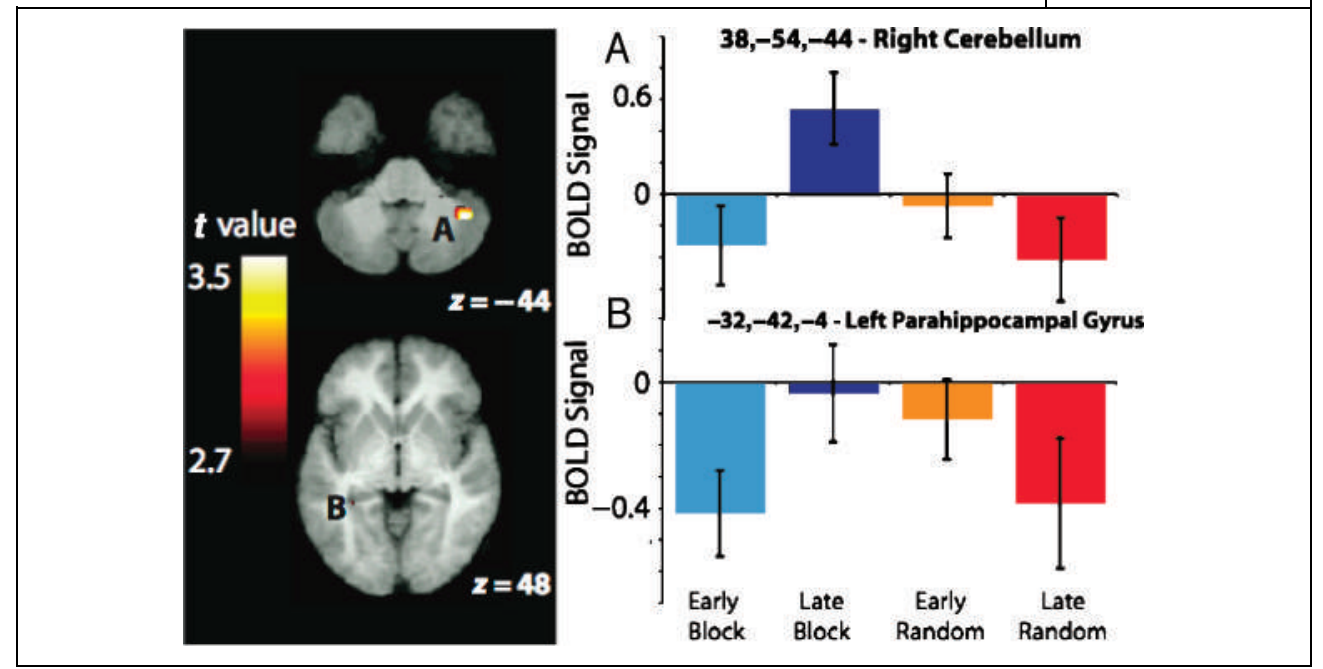

The most substantial empirical observation made by the present study is that differences in the training schedule between the blocked and random groups lead to recruitment of distinct neural substrates. This was true for both sequence preparation and task execution processes. As learning took place, individuals who practiced the task according to a random schedule showed increased activity in premotor and motor cortices during response preparation, suggesting that these participants were preparing each movement in advance more than the block practicing participants were. Because the random group went on to demonstrate better performance at retention testing, the findings suggest that the additional recruitment of premotor and motor areas by the end of training could play a central role in enhanced learning. During actual movement execution, the random practice structure resulted in increased activity in the SFG and MFG, whereas the block practice schedule led to widespread recruitment of motor preparatory and execution areas, including the superior parietal lobule, the dorsal premotor cortex, and the midline cerebellum. To best characterize how these imaging findings advance our knowledge of the CI phenomenon, it is useful to consider the findings for the random and block groups individually.

As training progressed, the random group showed increasing activity in the bilateral sensorimotor cortex and a lateral occipital region in the right hemisphere between early and late study periods compared to participants in the block group. Although the sensorimotor cortex is conventionally thought to be involved in movement execution, we can conclude that this activation is not an artifact from actual movement execution because we modeled the BOLD response from only the no-go trials where subjects simply rested after movement preparation. Moreover, increasing evidence from nonhuman primate studies (Lu \& Ashe, 2005; Georgopoulos, Taira, \& Lukashin, 1993; Kurata, 1993; Mushiake, Inase, \& Tanji, 1991; Alexander \& Crutcher, 1990) and human imaging and TMS work (Kansaku et al., 2005; Zang
Figure 9. Group differences of motor executioninteraction between increasing signal within the random group and decreasing signal within the block group. Differences were calculated from the first and the final $1 / 3$ of training trials, and are masked by regions that are active in all participants during movement time (Figure 5B). The left image is the left side of the brain. Threshold is $p<.005$, uncorrected, threshold $>3$ voxels. Statistical images are superimposed on a mean anatomic image of the study population.

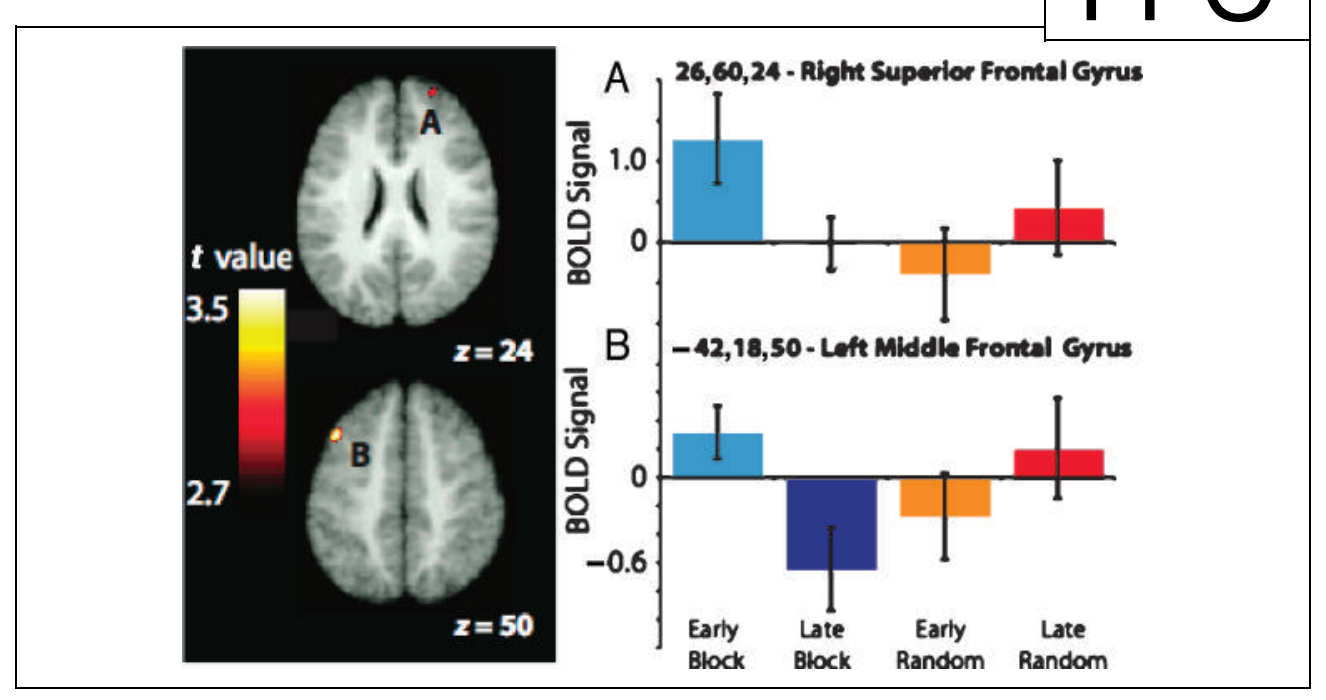


Table 4. Regions Showing Greater Activity in the Between-Group Comparisons for Movement Execution

\begin{tabular}{|c|c|c|c|c|c|c|c|}
\hline \multirow[b]{2}{*}{ Region } & \multirow[b]{2}{*}{$B A$} & \multicolumn{3}{|c|}{ MNI Coordinates } & \multirow[b]{2}{*}{ Functional Name } & \multirow[b]{2}{*}{$t$} & \multirow[b]{2}{*}{$p$} \\
\hline & & $x$ & $y$ & $z$ & & & \\
\hline \multicolumn{8}{|c|}{ Movement Time: Random Increasing (Early > Late) $>$ Block Increasing (Early $>$ Late) } \\
\hline L Superior/middle frontal gyrus & 6 & -42 & 18 & 50 & SFG/MFG & 3.62 & .001 \\
\hline R Superior frontal gyrus & 10 & 26 & 60 & 24 & SFG & 3.06 & .003 \\
\hline \multicolumn{8}{|c|}{ Movement Time: Block Increasing (Early > Late) $>$ Random Increasing (Early $>$ Late) } \\
\hline L Insula & 13 & -40 & -18 & 12 & & 5.78 & $<.001$ \\
\hline L Superior parietal lobule & 7 & -16 & -54 & 64 & SPL & 5.64 & $<.001$ \\
\hline R Inferior parietal sulcus & 7 & 16 & -48 & 50 & IPS & 4.77 & $<.001$ \\
\hline L Cingulate sulcus & 24 & -14 & 2 & 40 & & 4.75 & $<.001$ \\
\hline L Inferior postcentral sulcus & 1 & -60 & -22 & 42 & & 4.60 & $<.001$ \\
\hline R Postcentral gyrus & 2 & 48 & -14 & 12 & & 4.44 & $<.001$ \\
\hline L Superior frontal gyrus & 6 & -26 & -4 & 56 & SFG & 4.41 & $<.001$ \\
\hline L Inferior parietal lobule & 40 & -32 & -48 & 26 & IPL & 4.39 & $<.001$ \\
\hline R Fusiform gyrus & 37 & 30 & -48 & -10 & & 4.33 & $<.001$ \\
\hline L Angular gyrus & 39 & -30 & -68 & 22 & & 4.19 & $<.001$ \\
\hline R Cingulate cortex & 23 & 14 & -30 & 30 & CMA & 3.85 & $<.001$ \\
\hline R Cerebellum & & 12 & -44 & -36 & & 3.84 & $<.001$ \\
\hline L Superior temporal gyrus & 41 & -48 & -48 & 12 & STG & 3.83 & $<.001$ \\
\hline $\mathrm{R}$ Inferior frontal gyrus & 44 & 64 & 6 & 18 & IFG/PMv & 3.72 & .001 \\
\hline L Intraparietal sulcus & 7 & -42 & -46 & 50 & IPS & 3.67 & .001 \\
\hline R Parahippocampal cortex & $35 / 36$ & 14 & -38 & -2 & & 3.64 & .001 \\
\hline L Parahippocampal cortex & $35 / 36$ & -12 & -40 & -2 & & 3.63 & .001 \\
\hline R Occipito-temporal cortex & 37 & 46 & -64 & -4 & MT/V5 & 3.57 & .001 \\
\hline L Cingulate sulcus & 24 & -12 & -22 & 50 & CMA & 3.54 & .001 \\
\hline R Middle temporal gyrus & 22 & 30 & -58 & 30 & MTG & 3.54 & .001 \\
\hline L Superior frontal gyrus & 6 & -10 & -8 & 60 & SMA & 3.53 & .001 \\
\hline R Hippocampus & & 38 & -10 & -28 & & 3.53 & .001 \\
\hline L Insula & 13 & -26 & 6 & 20 & & 3.46 & .001 \\
\hline R Supramarginal gyrus & 40 & 54 & -32 & 38 & & 3.46 & .001 \\
\hline R Posterior middle temporal gyrus & 37 & 52 & -68 & 16 & MTG & 3.39 & .001 \\
\hline L Inferior temporal gyrus & 20 & -40 & -4 & -30 & & 3.36 & .001 \\
\hline $\mathrm{R}$ Anterior cingulate cortex & 24 & 16 & 30 & 14 & & 3.28 & .002 \\
\hline R Lingual gyrus & 37 & 24 & -66 & -6 & & 3.24 & .002 \\
\hline R Middle frontal gyrus & 6 & 46 & -8 & 40 & MFG & 3.24 & .002 \\
\hline L Insula & 13 & -38 & 22 & 6 & & 3.22 & .002 \\
\hline R Lateral cerebellum & & 42 & -54 & -40 & & 3.19 & .002 \\
\hline R Postcentral gyrus & 1 & 30 & -46 & 66 & $\mathrm{~S} 1$ & 3.18 & .002 \\
\hline L Fusiform gyrus & 19 & -44 & -70 & -22 & & 3.16 & .002 \\
\hline
\end{tabular}


Table 4. (continued)

\begin{tabular}{|c|c|c|c|c|c|c|c|}
\hline \multirow[b]{2}{*}{ Region } & \multirow[b]{2}{*}{$B A$} & \multicolumn{3}{|c|}{ MNI Coordinates } & \multirow[b]{2}{*}{ Functional Name } & \multirow[b]{2}{*}{$t$} & \multirow[b]{2}{*}{$p$} \\
\hline & & $x$ & $y$ & $z$ & & & \\
\hline L Superior parietal lobule & 7 & -22 & -66 & 44 & SPL & 3.10 & .002 \\
\hline $\mathrm{R}$ Thalamus & & 24 & -30 & -2 & & 3.08 & .002 \\
\hline L Primary motor cortex & 4 & -32 & -26 & 54 & M1 & 3.03 & .003 \\
\hline L Superior temporal gyrus & 22 & -32 & -34 & 18 & STG & 3.01 & .003 \\
\hline L Postcentral gyrus & 7 & -60 & -38 & 32 & & 3.01 & .003 \\
\hline L Fusiform gyrus & 37 & -38 & -22 & -22 & & 3.00 & .003 \\
\hline R Precentral gyrus & 6 & 46 & -4 & 54 & & 2.97 & .003 \\
\hline R Postcentral gyrus & 1 & 58 & -22 & 54 & & 2.97 & .003 \\
\hline $\mathrm{R}$ Cingulate gyrus & 31 & 14 & -20 & 48 & CMA & 2.95 & .003 \\
\hline R Inferior parietal lobule & 7 & 60 & -32 & 52 & IPL & 2.94 & .004 \\
\hline R Postcentral gyrus & 1 & 50 & -24 & 62 & & 2.93 & .004 \\
\hline L Intraparietal sulcus & 7 & -46 & -48 & 40 & IPS & 2.93 & .004 \\
\hline R Supramarginal gyrus & 40 & 68 & -36 & 34 & & 2.91 & .004 \\
\hline R Superior temporal gyrus & $41 / 42$ & 58 & -24 & 10 & STG & 2.90 & .004 \\
\hline R Temporo-occipital junction & 19 & 42 & -62 & 12 & $\mathrm{MT} / \mathrm{V} 5$ & 2.90 & .004 \\
\hline R Inferior occipital gyrus & 18 & 34 & -78 & -8 & & 2.89 & .004 \\
\hline L Parahippocampal gyrus & 36 & -24 & -34 & -20 & & 2.89 & .004 \\
\hline $\mathrm{R}$ Posterior cingulate area & 23 & 12 & -54 & 2 & & 2.86 & .004 \\
\hline $\mathrm{R}$ Uncus & 35 & 24 & 6 & -22 & & 2.85 & .004 \\
\hline L Inferior temporal gyrus & 37 & -56 & -52 & -14 & ITG & 2.84 & .004 \\
\hline
\end{tabular}

Significance at all sites was tested by an independent-samples $t$ test on beta values averaged over each voxel in the cluster, uncorrected at $p<.005$

et al., 2003; Grafton et al., 1998, 2002; Richter, Andersen, Georgopoulos, \& Kim, 1997) indicate that this cortical region is involved in the preparation of single and sequential movements. The finding of left hemisphere primary motor activation in a task where individuals are preparing to make a movement with their left hand is also in agreement with prior research implicating a role for this cortical region in both contralateral and ipsilateral hand movements (Kansaku et al., 2005; Cisek, Crammond, \& Kalaska, 2003; Schluter, Krams, Rushworth, \& Passingham, 2001; Shibasaki et al., 1993). The area of increasing signal in the right lateral occipital cortex is in line with prior EEG work on motor intention, which has demonstrated activation of this area in association with activation of the fronto-parietal attention network while performing a motor task (Praamstra, Boutsen, \& Humphreys, 2005).

In terms of neural activity in the random group during movement execution, the random practice structure resulted in increasing activity in the SFG and MFG. Work with nonhuman primates and event-related potentials and fMRI studies with humans implicate these frontal cortical areas as a key node for executive control and action set selection during motor tasks (for a review, see Rushworth et al., 2004). Several event-related potential experiments investigating components of motor task switching have demonstrated SFG activation when responses must actually change between trials, and less activity in this area when changes in task structure did not require new motor responses (Rushworth, Passingham, \& Nobre, 2002, 2005). Evidence from a study applying TMS over the SFG additionally supports the idea that the SFG is critically involved in switching motor responses between trials (Rushworth, Hadland, et al., 2002). In this combined fMRI/TMS study, rTMS over the SFG resulted in slower responses when subjects were instructed to switch the rule to which they were responding to an array of visual stimuli, but the rTMS did not lengthen response time when the task instructions remained the same, or when it was applied over several different control regions. Taken together, prior evidence implicates a role for SFG activity when action sets are initiated or switched, and it appears that the random practice structure could be modulating activity within this executive 
Figure 10. Group differences of motor executioninteraction between increasing signal within the block group and decreasing signal within the random group. Differences were calculated from the first and the final $1 / 3$ of training trials, and are masked by regions that are active in all participants during movement time (Figure 5B). The left image is the left side of the brain. Threshold is $p<.005$, uncorrected, threshold $>$ 3 voxels. Statistical images are superimposed on a mean anatomic image of the study population.

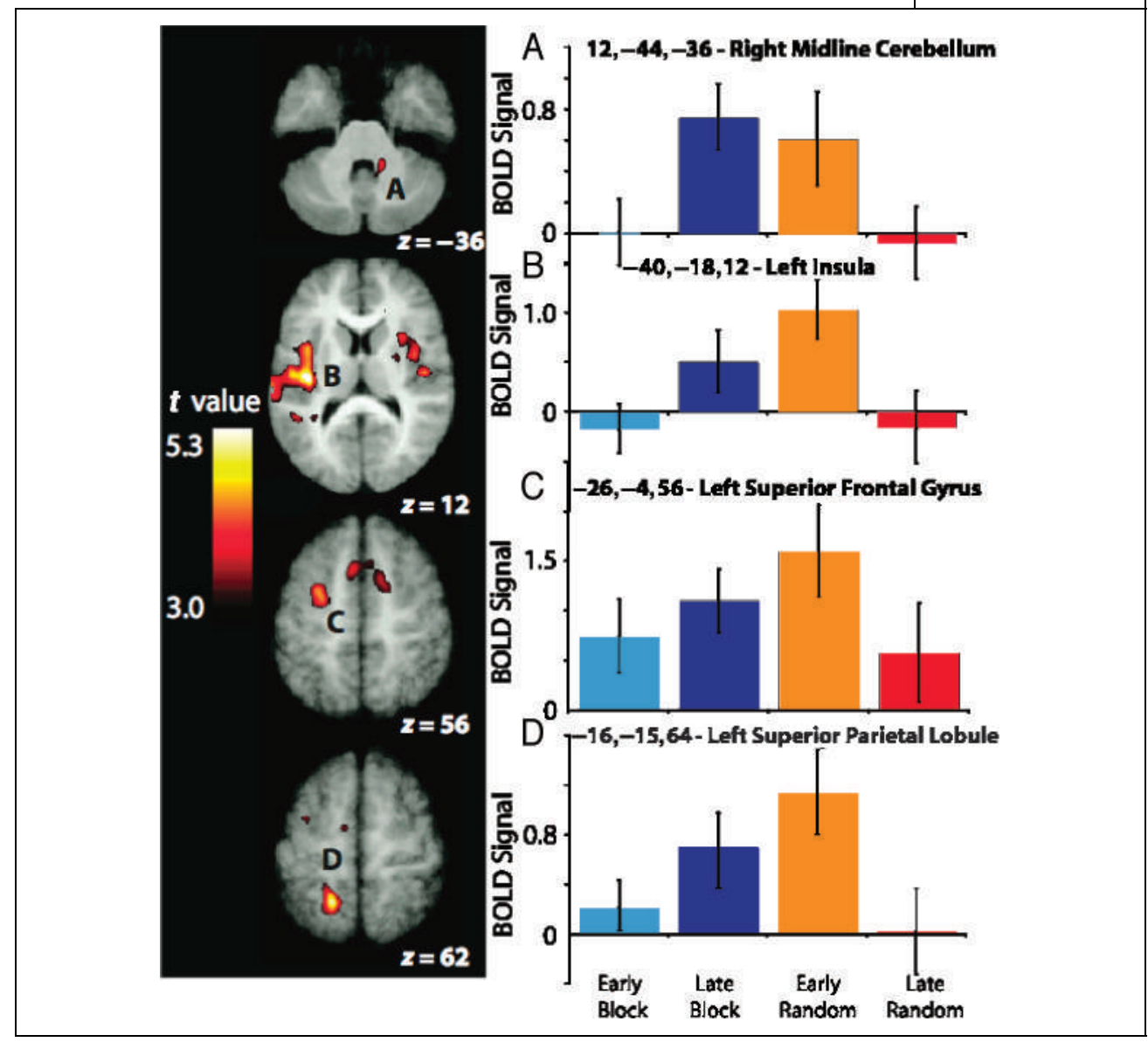

function circuit during movement execution, which might be leading to later performance benefits.

For movement execution, an interesting and complex pattern of increasing activity was observed in the block group compared to the random group. Specifically, an extensive network of regions involved in movement preparation and execution demonstrated increasing activity across time. This general finding is in accord with basic motor programming literature (Klapp, 1996), and with what other CI researchers have suggested in the past; namely, that participants in the block group wait for the go cue to perform some elements of movement reconstruction (Wright, Black, Immink, Brueckner, \& Magnuson, 2004). This idea is supported by both our imaging evidence, which demonstrate areas recruited in motor sequence reconstruction, and the behavioral data, which show that participants in the block group take longer to execute the movement sequences, although this difference is not significant (refer to Figure 4). The four areas that were the focus of ROI analyses, the right midline cerebellum, left insula, left SFG, and left SPL, were among the most significant activations, and those most critically involved in movement preparation and execution.

The activity seen in the midline cerebellum fits well with past imaging work that has associated this area with execution of hand and finger movements (Grodd, Hulsmann, Lotze, Wildgruber, \& Erb, 2001). In terms of the large area of insula activation observed, this activity might be related to general task-set maintenance across training, and less specific to the motor parameters of this task. Evidence for this idea comes from a study that reanalyzed data from eight mixed block/eventrelated fMRI designs using 10 different tasks, including motor timing, semantic judgments, and object naming (Dosenbach et al., 2006). These authors suggest that parts of the anterior insula are involved in network of regions that are generally active during the implementation of goal-directed task sets. The left SFG, specifically the dorsal premotor region of this area, has been shown to be associated with sequence retrieval, and to some extent, sequence execution, by myriad studies (Ashe et al., 2006; Kansaku et al., 2005; Bischoff-Grethe et al., 2004; Grafton et al., 1998, 2002; Honda et al., 1998; Nakamura, Sakai, \& Hikosaka, 1998; Jenkins et al., 1994). Another cortical region demonstrating greater activity during movement execution in participants assigned to the block practice group was a region in the left superior parietal cortex/lobe (SPL). An elegant monkey study by Gemba, Matsuura-Nakao, and Matsuzaki (2004) demonstrated that readiness potentials in the posterior parietal cortex are seen at approximately $1 \mathrm{sec}$ before the onset 
of self-paced movements of the hands and other body parts. In humans, precedent for SPL involvement in movement preparation, redirection, and more generally, motor attention has been demonstrated by a number of functional neuroimaging studies (Rushworth, JohansenBerg, Gobel, \& Devlin, 2003; Thoenissen, Zilles, \& Toni, 2002; Rushworth, Ellison, \& Walsh, 2001; Kalaska \& Crammond, 1995). A common finding among these studies is that the superior parietal cortex in the left hemisphere plays the dominant role in motor attention, which might be assisting participants in the block group with movement preparation and execution during the movement time period.

In the context of theoretical accounts that attempt to explain the CI effect, we believe our findings are in accord with the reconstruction hypothesis of CI. The basic premise of this hypothesis is that impaired initial performance leading to performance benefits on retest tasks is caused by effortful purging and reconstructing of task information with each trial (Jacoby, 1978). In the present study, the imaging data demonstrating greater activity within sensorimotor areas during movement preparation suggest that participants in the random group engage in more movement preparation than their block practice counterparts. The increased activity within the SFG/MFG during movement execution suggests that participants in the random group are recruiting additional higher cognitive areas involved in executive control by the end of training, another factor that can facilitate successful action retrieval and performance. It must be acknowledged that this pattern of results (i.e., more activity in M1 during movement preparation and more activity in the SFG/MFG during movement execution) is, in some ways, counterintuitive. Although it is difficult at this stage to fully explain the observed pattern of findings, several of the imaging studies discussed above provide some measure of support for the present results. Further evidence for the importance of M1 activity during movement preparation (in addition to movement execution) comes from work performed with monkeys on sequential skill representation in M1 (Matsuzaka et al., 2007; Lu \& Ashe, 2005). These studies demonstrated that over long-term motor sequence learning, M1 neurons encode sequence information both in anticipation of a response, as well as during response execution. The findings we report in the present study are consistent with the role of M1 in response anticipation.

Future research could investigate the theoretical underpinnings of the CI phenomenon further to determine whether the reconstruction hypothesis is specific to motor learning. Also, at this stage, it is an unproven inference that the differences in brain activity between the two groups at the end of training caused performance differences between the two groups on the retention task. Although the design of the present study precludes the possibility of conducting in-depth corre- lational analyses between the neural activity profile at the end of training and performance scores on the retest measure, this is something that future studies can explore further.

There are several limitations to our study that warrant discussion. The first is the fact that we have presented imaging findings for only the first half of the CI story. The great paradox of CI is that participants' performance during training is substantially different from their performance on retention tests. In pilot versions of the present study, we imaged participants' brains while they were performing retest sessions 24 hours after initial training in the scanner. However, we encountered problems with both the behavioral and imaging data for this procedure. In order to collect enough imaging data for meaningful analyses, participants needed to perform more retest trials than are usually administered in CI retests. This is problematic because, with a large number of trials, the retention test is transformed into a follow-up training session and the groups' performance begins to converge. Future work on the neural substrates of CI could endeavor to find a way to meaningfully explore the neural substrates of the retention and transfer stages of this phenomenon.

Another limitation of this study is the use of a betweensubjects design. To our knowledge, no studies have yet been published that demonstrate within-subjects CI, because CI has traditionally been implemented and studied as a between-group phenomenon (Lee \& Simon, 2004; Magill \& Hall, 1990). However, the power of inferences that can be drawn from a between-groups fMRI investigation can be amplified by using a within-groups design. To that end, it would be edifying to see if the same dissociable neural substrates for blocked and random practice that were observed in the present study would manifest in a within-subjects fMRI CI study.

The differences in study time between the groups during the early phase are another potential limitation. Study time on each trial was modeled in the duration parameter of each event in the design matrix, but the limits of our knowledge of precisely how hemodynamic responses track neural firing patterns during study time means that this model may not be entirely accurate. Thus, our analyses that include the early phase of the data must be understood in the context of these uncertainties. To address this, we also analyzed differences of brain activity for just the late phase of the study time data, where there were no group differences of ST duration. We did find one common region between the interaction contrast and the direct contrast of just the late period of study time between groups in M1 in the right hemisphere. This suggests to us that the effect in this area is not due to differences in study time and is instead a consequence of structuring practice according to a random schedule. This finding of persistent M1 activity in the randomly practiced group is in accord with prior imaging work on motor sequence learning with the left 
hand when task demands are especially challenging (Grafton et al., 2002).

In summary, the present results demonstrate the presence of distinct patterns of neural activity between groups at the end of training depending on the assigned practice schedule. We interpret these differences of neural activity to be related to participants' subsequent behavioral performance on retention and transfer tasks. Because our CI-related effects were primarily located in sensorimotor cortical areas, this leads us to believe that $\mathrm{CI}$ is a modality-specific effect. We speculate that because the benefits of $\mathrm{CI}$ in the present motor task are manifest in motor areas, then CI effects observed in a cognitive task would be manifest in changes in the cortex more involved in cognition. Future studies can investigate this issue further. Future work could also further explore the practical applications of the neural substrates of CI by quantifying changes in neural activity when CI paradigms are applied in sports coaching, physical therapy, and occupational therapy contexts.

\section{Acknowledgments}

This study was supported by a Science of Learning Center grant from the National Science Foundation and PHS grant NS33504. We thank Carolee Winstein for the insight and encouragement to develop this study, Roger Newman-Norlund for programming assistance, Antonia Hamilton for analyses assistance, and Nick Wymbus, Nichola Rice, David Wright, and an anonymous reviewer for helpful comments on earlier versions of this manuscript.

Reprint requests should be sent to Scott T. Grafton, Center for Cognitive Neuroscience, 6162 Moore Hall, Dartmouth College, Hanover, NH 03755, or via e-mail: scott.t.grafton@ dartmouth.edu.

\section{REFERENCES}

Alexander, G. E., \& Crutcher, M. D. (1990). Neural representations of the target (goal) of visually guided arm movements in three motor areas of the monkey. Journal of Neurophysiology, 64, 164-178.

Ashe, J., Lungu, O. V., Basford, A. T., \& Lu, X. (2006). Cortical control of motor sequences. Current Opinion in Neurobiology, 16, 213-221.

Battig, W. F. (1972). Intratask interference as a source of facilitation in transfer and retention. In R. F. Thompson \& J. F. Voss (Eds.), Topics in learning and performance. New York: Academic Press.

Ben-Shaul, Y., Drori, R., Asher, I., Stark, E., Nadasdy, Z., \& Abeles, M. (2004). Neuronal activity in motor cortical areas reflects the sequential context of movement. Journal of Neurophysiology, 91, 1748-1762.

Bischoff-Grethe, A., Goedert, K. M., Willingham, D. T., \& Grafton, S. T. (2004). Neural substrates of response-based sequence learning using fMRI. Journal of Cognitive Neuroscience, 16, 127-138.

Brady, F. (1997). Contextual interference and teaching golf skills. Perceptual and Motor Skills, 84, 347-350.

Cisek, P., Crammond, D. J., \& Kalaska, J. F. (2003). Neural activity in primary motor and dorsal premotor cortex in reaching tasks with the contralateral versus ipsilateral arm. Journal of Neurophysiology, 89, 922-942.

de Croock, M. B. M., van Merrienboer, J. G. J., \& Paas, F. G. W. C. (1998). High versus low contextual interference in simulation-based training of troubleshooting skills: Effects on transfer performance and invested mental effort. Computers in Human Behavior, 14, 249-267.

Diedrichsen, J., Grafton, S., Albert, N., Hazeltine, E., \& Ivry, R. B. (2006). Goal-selection and movement-related conflict during bimanual reaching movements. Cerebral Cortex, 16, 1729-1738.

Diedrichsen, J., Werner, S., Schmidt, T., \& Trommershauser, J. (2004). Immediate spatial distortions of pointing movements induced by visual landmarks. Perception \& Psychophysics, 66, 89-103.

Dosenbach, N. U., Visscher, K. M., Palmer, E. D., Miezin, F. M., Wenger, K. K., Kang, H. C., et al. (2006). A core system for the implementation of task sets. Neuron, 50, 799-812.

Forkstam, C., \& Petersson, K. M. (2005). Towards an explicit account of implicit learning. Current Opinion in Neurology, 18, 435-441.

Friston, K. J., Fletcher, P., Josephs, O., Holmes, A., Rugg, M. D., \& Turner, R. (1998). Event-related fMRI: Characterizing differential responses. Neuroimage, 7, 30-40.

Gemba, H., Matsuura-Nakao, K., \& Matsuzaki, R. (2004). Preparative activities in posterior parietal cortex for self-paced movement in monkeys. Neuroscience Letters, 357, 68-72.

Georgopoulos, A. P., Taira, M., \& Lukashin, A. (1993). Cognitive neurophysiology of the motor cortex. Science, 260, 47-52.

Goodwin, J. E., \& Meeuwsen, H. J. (1996). Investigation of the contextual interference effect in the manipulation of the motor parameter of over-all force. Perceptual and Motor Skills, 83, 735-743.

Grafton, S. T., Hazeltine, E., \& Ivry, R. B. (1998). Abstract and effector-specific representations of motor sequences identified with PET. Journal of Neuroscience, 18, 9420-9428.

Grafton, S. T., Hazeltine, E., \& Ivry, R. B. (2002). Motor sequence learning with the nondominant left hand. A PET functional imaging study. Experimental Brain Research, 146, 369-378.

Grodd, W., Hulsmann, E., Lotze, M., Wildgruber, D., \& Erb, M. (2001). Sensorimotor mapping of the human cerebellum: fMRI evidence of somatotopic organization. Human Brain Mapping, 13, 55-73.

Honda, M., Deiber, M. P., Ibanez, V., Pascual-Leone, A., Zhuang, P., \& Hallett, M. (1998). Dynamic cortical involvement in implicit and explicit motor sequence learning. A PET study. Brain, 121, 2159-2173.

Immink, M., \& Wright, D. L. (1998). Contextual interference: A response planning account. Quarterly Journal of Experimental Psychology, 51A, 735-754.

Jacoby, L. L. (1978). On interpreting the effects of repetition: Solving a problem versus remembering a solution. Journal of Verbal Learning and Verbal Behavior, 17, 649-667.

Jenkins, I. H., Brooks, D. J., Nixon, P. D., Frackowiak, R. S., \& Passingham, R. E. (1994). Motor sequence learning: A study with positron emission tomography. Journal of Neuroscience, 14, 3775-3790.

Johnson-Frey, S. H., Newman-Norlund, R., \& Grafton, S. T. (2005). A distributed left hemisphere network active during planning of everyday tool use skills. Cerebral Cortex, 15, 681-695.

Kalaska, J. F., \& Crammond, D. J. (1995). Deciding not to GO: Neuronal correlates of response selection in a 
$\mathrm{GO} / \mathrm{NOGO}$ task in primate premotor and parietal cortex. Cerebral Cortex, 5, 410-428.

Kansaku, K., Muraki, S., Umeyama, S., Nishimori, Y., Kochiyama, T., Yamane, S., et al. (2005). Cortical activity in multiple motor areas during sequential finger movements: An application of independent component analysis. Neuroimage, 28, 669-681.

Keele, S. W. (1986). Motor control. In K. R. Boll, L. Kaufman, \& J. P. Thomas (Eds.), Handbook of perception and buman performance: Vol. 2. Cognitive processes and performance (pp. 1-60). New York: Wiley.

Kennerley, S. W., Sakai, K., \& Rushworth, M. F. (2004). Organization of action sequences and the role of the pre-SMA. Journal of Neurophysiology, 91, 978-993.

Klapp, S. T. (1996). Reaction time analysis of central motor control. In H. N. Zelaznik (Ed.), Advances in motor learning and control (pp. 13-34). Champaign: Human Kinetics.

Kurata, K. (1993). Premotor cortex of monkeys: Setand movement-related activity reflecting amplitude and direction of wrist movements. Journal of Neurophysiology, 69, 187-200.

Lee, T. D., \& Magill, R. A. (1983). The locus of contextual interference in motor-skill acquisition. Journal of Experimental Psychology: Learning, Memory, and Cognition, 9, 730-746.

Lee, T. D., Magill, R. A., \& Weeks, D. J. (1985). Influence of practice schedule on testing schema theory predictions in adults. Journal of Motor Behavior, 17, 283-299.

Lee, T. D., \& Simon, D. A. (2004). Contextual interference. In A. M. Williams \& N. J. Hodges (Eds.), Skill acquisition in sport: Research, theory and practice (pp. 29-44). London: Routledge.

Li, Y., \& Wright, D. L. (2000). An assessment of the attention demands during random- and blocked-practice schedules. Quarterly Journal of Experimental Psychology, Series A, Human Experimental Psychology 53, 591-606.

Lu, X., \& Ashe, J. (2005). Anticipatory activity in primary motor cortex codes memorized movement sequences. Neuron, 45, 967-973.

Magill, R. A., \& Hall, K. G. (1990). A review of the contextual interference effect in motor skill acquisition. Human Movement Science, 9, 241-289.

Matsuzaka, Y., Picard, N., \& Strick, P. L. (2007). Skill representation in the primary motor cortex after long-term practice. Journal of Neurophysiology, 97, 1819-1832.

Muellbacher, W., Ziemann, U., Wissel, J., Dang, N., Kofler, M., Facchini, S., et al. (2002). Early consolidation in human primary motor cortex. Nature, 415, 640-644.

Mushiake, H., Inase, M., \& Tanji, J. (1991). Neuronal activity in the primate premotor, supplementary, and precentral motor cortex during visually guided and internally determined sequential movements. Journal of Neurophysiology, 66, 705-718.

Nakamura, K., Sakai, K., \& Hikosaka, O. (1998). Neuronal activity in medial frontal cortex during learning of sequential procedures. Journal of Neurophysiology, 80, 2671-2687.

Oldfield, R. C. (1971). The assessment and analysis of handedness: The Edinburgh inventory. Neuropsychologia, 9, 97-113.

Ollis, S., Button, C., \& Fairweather, M. (2005). The influence of professional expertise and task complexity upon the potency of the contextual interference effect. Acta Psychologica (Amsterdam), 118, 229-244.

Passingham, R. E., Toni, I., \& Rushworth, M. F. (2000). Specialisation within the prefrontal cortex: The ventral prefrontal cortex and associative learning. Experimental Brain Research, 133, 103-113.

Praamstra, P., Boutsen, L., \& Humphreys, G. W. (2005). Frontoparietal control of spatial attention and motor intention in human EEG. Journal of Neurophysiology, 94, 764-774.

Richter, W., Andersen, P. M., Georgopoulos, A. P., \& Kim, S. G. (1997). Sequential activity in human motor areas during a delayed cued finger movement task studied by time-resolved fMRI. NeuroReport, 8, 1257-1261.

Robertson, E. M., Press, D. Z., \& Pascual-Leone, A. (2005). Off-line learning and the primary motor cortex. Journal of Neuroscience, 25, 6372-6378.

Rosenbaum, D. A. (1991). Human motor control. San Diego: Academic Press.

Rushworth, M. F., Ellison, A., \& Walsh, V. (2001). Complementary localization and lateralization of orienting and motor attention. Nature Neuroscience, 4, 656-661.

Rushworth, M. F., Hadland, K. A., Paus, T., \& Sipila, P. K. (2002). Role of the human medial frontal cortex in task switching: A combined fMRI and TMS study. Journal of Neurophysiology, 87, 2577-2592.

Rushworth, M. F., Johansen-Berg, H., Gobel, S. M., \& Devlin, J. T. (2003). The left parietal and premotor cortices: Motor attention and selection. Neuroimage, 20(Suppl. 1), S89-S100.

Rushworth, M. F., Passingham, R. E., \& Nobre, A. C. (2002). Components of switching intentional set. Journal of Cognitive Neuroscience, 14, 1139-1150.

Rushworth, M. F., Passingham, R. E., \& Nobre, A. C. (2005). Components of attentional set-switching. Experimental Psychology, 52, 83-98.

Rushworth, M. F., Walton, M. E., Kennerley, S. W., \& Bannerman, D. M. (2004). Action sets and decisions in the medial frontal cortex. Trends in Cognitive Sciences, 8, 410-417.

Schluter, N. D., Krams, M., Rushworth, M. F., \& Passingham, R. E. (2001). Cerebral dominance for action in the human brain: The selection of actions. Neuropsychologia, 39, $105-113$.

Schneider, V. I., Healy, A. F., Ericsson, K. A., \& Bourne, L. E. (1995). The effects of contextual interference on the acquisition and retention of logical rules. In A. F. Healy \& L. E. Bourne (Eds.), Learning and memory of knowledge and skills: Durability and specificity (pp. 95-131). Thousand Oaks, CA: Sage.

Shea, J. B., \& Morgan, R. L. (1979). Contextual interference effects on the acquisition, retention, and transfer of a motor skill. Journal of Experimental Psychology: Learning, Memory, and Cognition, 5, 179-187.

Shibasaki, H., Sadato, N., Lyshkow, H., Yonekura, Y., Honda, M., Nagamine, T., et al. (1993). Both primary motor cortex and supplementary motor area play an important role in complex finger movement. Brain, 116, 1387-1398.

Smith, P. J. (2002). Applying contextual interference to snowboarding skills. Perceptual and Motor Skills, 95, 999-1005.

Smith, P. J., \& Davies, M. (1995). Applying contextual interference to the Pawlata roll. Journal of Sports Science, 13, 455-462.

Smith, P. J., Gregory, S. K., \& Davies, M. (2003). Alternating versus blocked practice in learning a cartwheel. Perceptual and Motor Skills, 96, 1255-1264.

Talairach, J., \& Tournoux, P. (1988). Co-planar stereotaxic atlas of the buman brain. New York: Thieme.

Thoenissen, D., Zilles, K., \& Toni, I. (2002). Differential involvement of parietal and precentral regions in movement 
preparation and motor intention. Journal of Neuroscience, 22, 9024-9034.

Toni, I., Ramnani, N., Josephs, O., Ashburner, J., \& Passingham, R. E. (2001). Learning arbitrary visuomotor associations: Temporal dynamic of brain activity. Neuroimage, 14, 1048-1057.

Toni, I., Rushworth, M. F., \& Passingham, R. E. (2001). Neural correlates of visuomotor associations. Spatial rules compared with arbitrary rules. Experimental Brain Research, 141, 359-369.
Wright, D. L., Black, C. B., Immink, M. A., Brueckner, S., \& Magnuson, C. (2004). Long-term motor programming improvements occur via concatenation of movement sequences during random but not during blocked practice. Journal of Motor Behavior, 36, 39-50.

Zang, Y., Jia, F., Weng, X., Li, E., Cui, S., Wang, Y., et al. (2003). Functional organization of the primary motor cortex characterized by event-related fMRI during movement preparation and execution. Neuroscience Letters, 337, 69-72. 


\section{AUTHOR QUERIES}

\section{AUTHOR PLEASE ANSWER QUERIES}

During the preparation of your manuscript, the questions listed below arose. Kindly supply the necessary information.

1. Paragraph before the Results section: Please check if the minimum cluster size $643 \mathrm{~mm}$ should actually read $64 \mathrm{~mm} 3$.

2. Discussion section, paragraph 11: Please define ST in ST duration.

3. Table 4: Please check if inferior parietal sulcus and intraparietal sulcus is one and the same. They both use IPS as functional name.

3. Citation of Figure 5A and 5B was found in Figures 1-10 caption, however, no figure labels were found for Figure 5 image. Please confirm if the citation will be deleted or the image labels A and B will be added for Figure 5?

\section{END OF ALL QUERIES}

